\title{
Development of Eye-Blink and Face Corpora for Research in Human Computer Interaction
}

\author{
Emmanuel Jadesola Adejoke. \\ Dept. of Computer science \\ Bingham University \\ Nassarawa, Nigeria
}

\author{
Ibiyemi Tunji Samuel \\ Dept. of Electrical Engineering \\ University of Ilorin \\ Ilorin, Nigeria
}

\begin{abstract}
A major requirement in face recognition research and coded voluntary eye-blink based sign language communication research is a robust face and eye-blink image corpora. The effectiveness, confidence level, and acceptability level of developed algorithms for face recognition and eye-blink based sign language communication depends largely on availability of relevant corpora in these fields of international standard. The wave of security challenges with attendant wanton destruction to lives and properties particularly in our country makes deployment of appropriate information technology to curb it imperative. Hence, the motivation of this work in provision of face and eye-blink image corpora with local contents to serve as input dataset to our developed face recognition authenticated driven coded eye-blink triggered actionable alert.
\end{abstract}

Keywords-Face Recognition; Coded Voluntary Eye Blink; Sign Language Communication; Authenticated driven; corpora

\section{INTRODUCTION}

In recent years, there have been efforts to augment traditional Human Computer interfaces with intelligent interfaces that allow users to interact with the computer more naturally and effectively. The goal is to develop HCI that are more man-centered in communication than computercentered.[1] These are to respond to users natural communication cues such as gestures, facial expressions, gaze directions etc. and have been implemented in systems such as face recognition systems, eye blink recognition system, lip reading systems, voice recognition and translation systems and sign language recognition systems.

Although there are existing reliable methods of biometric personal identification such as finger print analysis, retina or iris scan they are yet to gain general acceptance from the public [2]. Facial recognition is chosen because it is a more passive and non-intrusive system of authentication also it is an identification technique that is closer to the way human beings recognize each other. With the current insecurity in worldwide a non-intrusive authentication system such as face recognition will be desirable where the people involved may not even be conscious of been captured.

Eye blink as a sign language, has for ages been a mode of communication for human beings, where information of different codes are being passed from one person to another. For security or emergency issues a real time eye blink communication would be a very reliable mode of communication for quick responses. It is reported that the eyes are directly connected to brain [3], they are the last part of our body on which we can lose control in man. For some persons, who are suffering from a brain-stem stroke, neuro-motor disability or due to any accident, the eyes are the only option for communication with the world. Blink of their eyes can be converted to vocabulary for such persons. But continuous monitoring and understanding is required to understand and communicate with them properly. This is a bit difficult for human to understand the meaning of those blinks continuously [4].

It is noticed that world-wide there are many computer users who, due to their physical condition, are not able to use their hands or any part of body. Most of them have good control of their eyes and therefore communication with the world is done solely using the movement of their eyes or eye lids. These eyes blink or eye lids movement can be replaced with mouse click functionality. Implementing this man centered communication mode(eye blink communication) to a computer system for responses in these types of situations is very timely. Hence the development of the face and eye blink corpora. Many face databases has been created and made available for use and research purposes but most if not all of them are for the white coloured man. In this paper is presented corpora for the black coloured man both of frontal still faces for face recognition and dynamic video streams for eye blink recognition is presented.

In this proposed system, a cctv colour camera captures video stream digital images fed into a module for detecting face in the input image and crop out the face image.

Due to variations in the size and illumination effects on the cropped faces both size (geometric) and illumination normalization is carried out on the cropped face. The normalized face is then passed to face recognition unit and the eye-blink recognition unit simultaneously.

On detection and decoding of eye blinks, the desired action is performed if and only if the person making the blinks is authorized to communicate with the machine. Authorization is based on face recognition. The actionable operation supported in this work is automatic telephone dialing of some selected phone numbers relaying pre-recorded 'Save-Our-Soul' message by person under security threat or by an incapacitated medical patient In the section two of this paper the data collection experiment is discussed, the section three describes the corpora creation process and how it can be accessed, and section four is the conclusion of the paper. 


\section{RELATED WORKS}

A summary of some existing face databases is given in [5], where the authors created a database of black faces used to benchmark algorithms developed for skin segmentation, face development and recognition under real life situation. The researchers present still frontal view black face database collected under controlled conditions for researches that require two dimension still face images. In [6] RPI ISL presents seven eye databases used primarily in eye detection, eye tracking and eye blink recognition. Existing eye blink databases capture only single eye blinks but this collection contains both single and double eye blinks to make room for more robust and extensive researches in eye blink recognition.[7] Presents the ZJU Eye blink video database which consists of 80 video clips of 20 individuals in the avi format. This was used in the development of an eye detector and eye blink detector using AdaBoost tools and recorded a $99.34 \%$ rate of eye detection and a $96 \%$ accuracy of eye blink detection. This newly created eye blink video corpora contains 90 video streams of four minutes each capturing both involuntary eye blinks and prompted single and double eye blinks for a wider scope of research in theses area.[8]The BioID Face Database is a real world condition database with varying illumination background and face size. The dataset consists of 1521 gray level images with a resolution of $384 \times 286$ pixels. Each one shows the frontal view of a face of one out of 23 different test persons. For comparison reasons the set also contains manually set eye positions. The images are stored in single files using the portable gray map (pgm) data format. The database is published to give all researchers working in the area of face detection the possibility to compare the quality of their face detection algorithms with others. A new corpora is being created to have black faces databases relevant to the international community to aid more researches in the fields of face detection, face recognition and eye blink recognition.

[9]The AT\&T laboratory created a database of faces that contains a set of face images taken between April 1992 and April 1994 at the lab. The database was used in the context of a face recognition project carried out in collaboration with the Speech, Vision and Robotics Group of the Cambridge University Engineering Department. There are ten different images of each of 40 distinct subjects. For some subjects, the images were taken at different times, varying the lighting, facial expressions (open / closed eyes, smiling / not smiling) and facial details (glasses / no glasses). All the images were taken against a dark homogeneous background with the subjects in an upright, frontal position (with tolerance for some side movement). The files are in PGM format, the size of each image is $92 \times 112$ pixels, with 256 grey levels per pixel. The images are organised in 40 directories (one for each subject), which have names of the form $\mathrm{sX}$, where $\mathrm{X}$ indicates the subject number (between 1 and 40). In each of these directories, there are ten different images of that subject, which have names of the form Y.pgm, where $\mathrm{Y}$ is the image number for that subject (between 1 and 10). The database can be retrieved from http://www.cl.cam.ac.uk/Research/DTG/attarchive/pub/data/at t faces.tar.Z as a 4.5Mbyte compressed tar file or from http://www.cl.cam.ac.uk/Research/DTG/attarchive/pub/data/at t faces.zip as a ZIP file of similar size. The database was utilized in [10].

\section{DATA COLLECTION EXPERIMENTS}

The corpora consist of only Africans between the ages of eight and forty five. A total of 123 male and 80 female fontal images were captured. It consists of mainly adults, only twelve children were captured, this is to ensure a large age variance. The pictures and streams were captured without glasses; only eight people were captured with plain glasses on. Of the eighty females captured, six of them had vials covering their hair to their bodies. The video streams are a total of four minutes each of static frontal recordings of involuntary and voluntary single and double eye blinks. The facial expression for both the pictures and the video is neutral. The pictures are stored in the BMP format, while the videos are stored in the AVI format. The picture frames from the video streams are stored in the BMP format.

\section{A. Data Collection Process}

A video recording cam-coder was used to capture the data for both still pictures and video clips. The choice of a camcoder over a web cam was to enable the researches get good quality pictures and video streams. A JVC camera model GZMG 275 was used with f-stop f/1.4, exposure time 1/25 seconds, ISO speed ISO-200 and EXIF version 0221.

The consideration of the site setting was for the camera to be four feet in front of the person to be captured with a white background. Where illumination was not sufficient, flood lights were made available. Figure 1 Depicts a picture of the data capture studio setup.

\section{B. Black Eye-Blink and Face Corpora (BEFC)}

In the BEFC a total of two hundred and three (203) people were captured. One hundred and twenty three (123) males and eighty (80) females. Five frontal view pictures were captured of each of people in jpeg format at a dimension of 1632 by 1224, horizontal and vertical resolution of $72 \mathrm{dpi}$ each, resolution unit of 2, bit dept 24 and color representation of

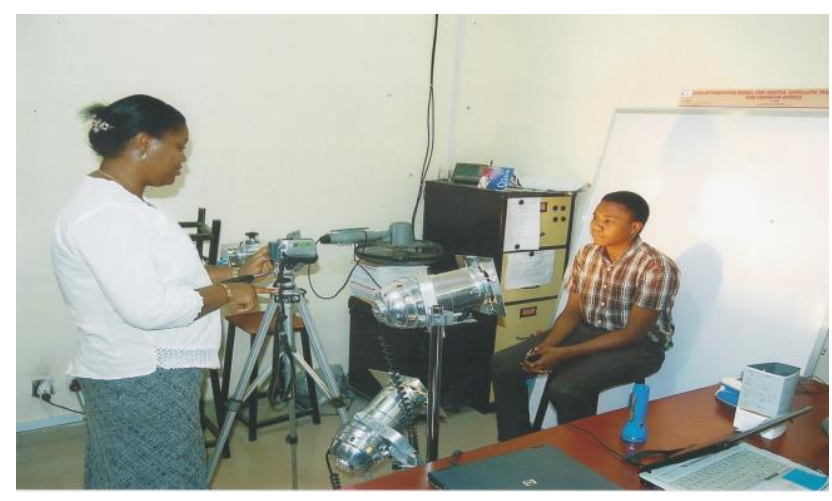

Fig. 1. Picture of Data Capture Studio Setup

sRGB. These were later converted into bmp format to ensure we have a loss less file. The bmp files from the pictures are of size 1632 by 1224 pixels each to be down sized to 640 by 480 pixels for a more reduce storage size and processing time. Each file is $900 \mathrm{~Kb}$ on disk and $24 \mathrm{bpp}$ (bit dept) each. Figure 2 shows sample faces in the face corpora. 
The video clips were made for four minutes each. They are frontal view video clips, the first two minutes was to capture involuntary eye blinks, in the third minute, prompted single eye blinks were taken and in the fourth minute prompted double eye blinks were taken. The video clips were taken in the .MOD format and later converted to the avi format because decoding program is easily done with the avi format. The .MOD files had a frame dimension of 720 by 576, frame rate of $25 \mathrm{fps}$, data rate $7700 \mathrm{kbps}$ and total bit rate 7956 kbps. The avi format has frames are of the dimension 720 by 576 pixels, data rate $1041 \mathrm{Kbps}$ (kilobytes per second), total bit rate of $1169 \mathrm{Kbps}$, frame rates 25 frames per second, bit rates $128 \mathrm{kbps}$, and of an average size of $33.7 \mathrm{Mb}$. A total of 90 videos clips were taken, 50 males and 40 females. The video clips were latter split into frames of bmp format of size $1.18 \mathrm{Mb}$ each, dimension 720 by 572, 24 bit colour. Eight of the people were captured with glasses to see how sensitive the system will be with glasses.

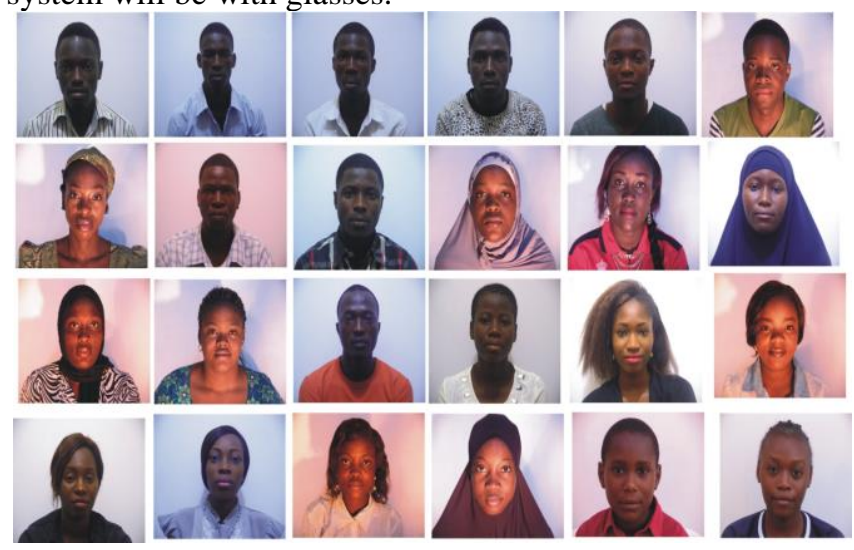

Fig. 2. Sample Faces in the Black Face Corpora

\section{Quantity and Quality of Collected Data}

The corpora stores one thousand one hundred and fifteen (1015) frontal view face pictures both of jpeg and bmp formats, ninety (90) video clips for four minutes each in the avi format and ninety folders of the frames of each video clip in the bmp format. The pictures are sharp, bright and distinct. There are some shadows at the background; the white background assumed various shades depending on the dominant color of the cloth of the person.

\section{CREATION AND ACCESS OF CORPORA}

\section{A. Description Of The Corpora Creation and Access Process}

The corpora are organised of four folders, the first folder contains 1165 pictures in the jpeg format, the second contains 1165 pictures in the bmp format, the third contains 90 video clips of four minutes each and the fourth contains 90 folder of the frames of each video clip in the bmp format.

The pictures have names of the form Fac_x_t. file extension. Where $\mathrm{x}$ stands for number numbering from 001223 and $\mathrm{t}$ stands for image number 1 to 5 for each subject. Figure 3 shows a sample of named pictures. The video streams have names of the form MOVxy.file extension, where $\mathrm{x}$ numbers from 1 to 90 and $\mathrm{y}$ is the number corresponding to the number of the picture of the person in the face database.
While the frame folders have names of the form MOVx , where $\mathrm{x}$ numbers from 1 to 90 . . The corpora can be accessed by contacting the first authors, the website where it can be uploaded is still under development and once it is ready it will be made public.

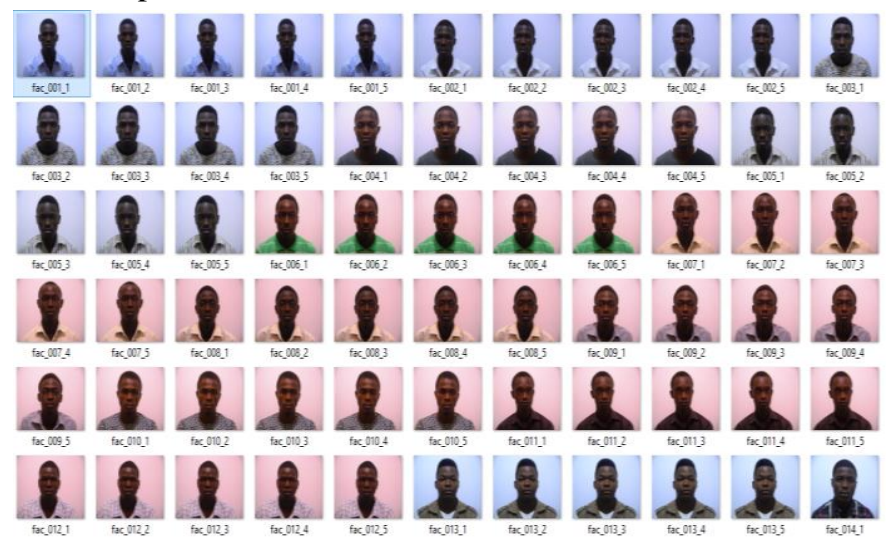

Fig. 3. Arrangement and Naming in The Black Face Corpora

\section{CONCLUSION}

This paper has discussed a new black face and eye blink corpora created for the development of a face recognition enabled by eye blink recognition system. The corpora are available for research as a contribution to the fields of HCI and bioinformatics for academic and non-commercial use especially for black faces. The system that will be developed will be applicable in the security system of the nation and also in the health sector

\section{REFERENCES}

[1] R. T. Narmadha, T. Mythili, R.T. Nivetha, " Real Time HCI Using Eye Blink Detection". Internationa Journal Of Computer Science And Mobile Computing, Vol. 3, Issue 12014

[2] M. Meenakshi, " Real-Time facial recognition system- Design, implementation and validation". Journal of signal processing theory and applications 2013.

[3] J.A. Emmanuel and T.S.Ibiyemi, "A Framework for a Face Recognition Enabled Eye Blink communication", in Book of Abstract, $1^{\text {st }}$ International Conference on Sustainable Development (ICSD 2015), Baze University, Abuja Nigeria.

[4] S. Priya, Parmar and Nehal Chitaliya, "Detect Eye blink using Motion analysis Method", International Journal of Emerging Technologies in Computational and Applied Sciences (IJETCAS), 2013

[5] J.A. Ojo and S.A. Adeniran, “ Colour Face Image Database for Skin Segmentation, Face detection, Recognition and Tracking for Black Faces Under Real Life Situations", International Journal of Image procesing(IJIP), vol 4 Issue 6, 2011

[6] http://www.ecse.rpi.edu/ cvrl/database/ISL_IR_Eye_Database.htm

[7] Gang Pan, Lin Sun, Zhaohui Wu, Shihong Lao, Eyeblink-based Antispoofing in Face Recognition from a Generic Webcamera, The 11th IEEE International Conference on Computer Vision (ICCV'07), Rio de Janeiro, Brazil, 2011.

[8] https://www.bioid.com/About/BioID-Face-Database

[9] www.cl.cam.ac.uk/research/dtg/attarchive/facedatabase.html

[10] Ferdinando Samaria, Andy Harter, Parameterisation of a Stochastic Model for Human Face Identification, Proceedings of 2nd IEEE Workshop on Applications of Computer Vision, Sarasota FL, December 1994. 